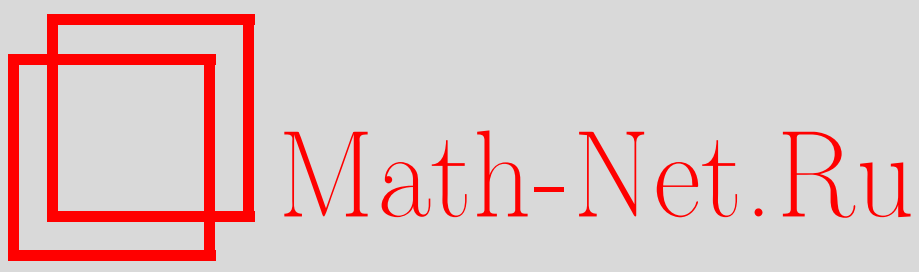

С. А. Кудрявцев, Дополнения на логповерхностях, Матем. сб., 2004, том 195, номер 6, 99-120

DOI: https://doi.org/10.4213/sm828

Использование Общероссийского математического портала Math-Net.Ru подразумевает, что вы прочитали и согласны с пользовательским соглашением

http://www . mathnet.ru/rus/agreement

Параметры загрузки:

IP : 3.85 .183 .62

26 апреля 2023 г., 15:32:46 
УДК 512.7

\title{
С.А. Кудрявцев
}

\section{Дополнения на логповерхностях}

\begin{abstract}
В настоящей работе уточняется основная индуктивная теорема о дополнениях на поверхностях и строятся модели для исключителњных логповерхностей дель Пецио с $\delta=0$.

Библиография: 18 названий.
\end{abstract}

\section{Введение}

Теория дополнений на алгебраических многообразиях была создана В. В. Шокуровым в работах [1], [2]. Она служит мощным инструментом для исследования алгебраических многообразий, экстремальных стягиваний и особенностей. Преимушество этой теории связано с тем, что понятие дополнения (под дополнением будет пониматься "хороший” дивизор в кратной антиканонической линейной системе) согласовано с логпрограммой минимальных моделей. Более того, дополнение обладает индуктивным свойством, т.е. проблема нахождения дополнения для $n$-мерного многообразия сводится к нахождению дополнения для $(n-1)$-мерного многообразия. По поводу теории дополнений для $n$-мерных многообразий см. работы [2]-[4]. Применение этой теории в трехмерном случае проиллюстрировано, например, в работах [5]-[8].

Итак, для эффективного исследования трехмерных стягиваний и особенностей важно полностью изучить логповерхности дель Пещц. Оставшаяся открытая двумерная проблема (в рамках теории дополнений) - это классификация исключительных логповерхностей дель Пещо. Исключительные логповерхности дель Пеццо $(S, D)$ делятся на три типа: $\delta(S, D)=0, \delta(S, D)=1$ и $\delta(S, D)=2$, где

$$
\delta(S, D)=\#\left\{E \mid E-\text { дивизор с дискрепантностью } a(E, D) \leqslant-\frac{6}{7}\right\} .
$$

Случаи $\delta(S, D)=1$ и $\delta(S, D)=2$ были разобраны в [2], [9]. Для изучения оставшегося случая $\delta(S, D)=0$ теорию дополнений на поверхностях необходимо применять в более широком классе коэффициентов. Поэтому она будет рассмотрена, когда все коэффициенты границы $D$ больше или равны $\frac{1}{2}$.

Один из основных результатов настоящей работы, приведенный в $\S 2,-$ это уточнение основной индуктивной теоремы о дополнениях на поверхностях. С помощью нее в $\S 4$ построены модели для исключительных логповерхностей дель Пещц

Работа выполнена при частичной поддержке Российского фонда фундаментальных исследований (грант № 02-01-00441), Программы поддержки ведущих научных школ РФ (грант № 00-15-96085) и фонда INTAS-OPEN (грант № 2000-269). 
с $\delta=0$ (см. определение 4.10). В $\S 3$ получена классификация исключительных нерациональных логповерхностей. Также в $\S 4$ изучен один тип исключительных логповерхностей с $\delta=0$ (см. теорема 4.3).

Автор признателен Ю.Г. Прохорову за ряд ценных советов.

\section{§ 1. Предварительные сведения и результаты}

Все многообразия рассматриваются над полем комплексных чисел $\mathbb{C}$. Основные определения, обозначения и понятия, использованные в статье, приведены в [3], [10].

ОПРЕДЕЛЕНИЕ 1.1. Положим $\Phi_{\mathrm{sm}}=\left\{1-\frac{1}{m} \mid m \in \mathbb{Z}_{>0} \cup\{\infty\}\right\}$ и $\Phi_{\mathrm{m}}=\Phi_{\mathrm{sm}} \cup$ $\left[\frac{6}{7}, 1\right]$. Коэффициент $d$ называется стандартньц, если $d \in \Phi_{\mathrm{sm}}$.

Положим $\mathbb{Z} /(n)=\left\{k / n \mid k \in \mathbb{Z}_{>0}\right\}$.

ОПРЕДЕЛЕНИЕ 1.2. Для $n \in \mathbb{N}$ положим

$$
\mathscr{P}_{n}=\{a \mid 0 \leqslant a \leqslant 1,\lfloor(n+1) a\rfloor \geqslant n a\} .
$$

Понятно, что

$$
\mathscr{P}_{n}=\{0\} \cup\left[\frac{1}{n+1}, \frac{1}{n}\right] \cup\left[\frac{2}{n+1}, \frac{2}{n}\right] \cup \cdots \cup\left[\frac{k}{n+1}, \frac{k}{n}\right] \cup \cdots \cup\left[\frac{n}{n+1}, 1\right] .
$$

ОПРЕДЕЛЕНИЕ 1.3. Определим множество $\Phi^{[a, b)}=\left(\Phi_{\mathrm{sm}} \cap[0, b)\right) \cup[a, b)$. При $a=(i-1) / i$ и $b=i /(i+1)$ положим $\Phi_{i}=\Phi^{[a, b)}$.

ОПРЕДЕЛЕНИЕ 1.4. Пара $\left(X, D=\sum d_{k} D_{k}\right)$ назьвается парой типа $\Phi_{i}$, если выполнены следующие три условия:

1) $d_{k} \in \Phi_{i}$ для всех $k$;

2) существует $j$ для которого $d_{j} \geqslant(i-1) / i$;

3) дивизор $K_{X}+D$ является $\frac{1}{i}$-логтерминальньм.

ОПРЕДЕЛЕНИЕ 1.5 . Пусть $(X / Z \ni P, D)$ - стягивание многообразий; $\mathbb{Q}$-дополнением для этого стягивания называется эффективный $\mathbb{Q}$-дивизор $D^{\prime}$ такой, что $D^{\prime} \geqslant D$, дивизор $K_{X}+D^{\prime}$ логканоничен и $K_{X}+D^{\prime} \sim_{\mathbb{Q}} 0$.

ОПРЕДЕЛЕНИЕ 1.6. Пусть $X$ - нормальное многообразие, а дивизор $D=S+B$ является субграницей, где $S$ - его целая эффективная часть и $\lfloor B\rfloor \leqslant 0$. Дивизор $K_{X}+D$ называется $n$-дополняемым, если сушествует $\mathbb{Q}$-дивизор $D^{+}$, называемый $n$-дополнением, который удовлетворяет следующим требованиям:

1) $n\left(K_{X}+D^{+}\right) \sim 0$

2) дивизор $K_{X}+D^{+}$логканоничен;

3) $n D^{+} \geqslant n S+\lfloor(n+1) B\rfloor$.

Если $X$ - полугладкое многообразие в коразмерности 1 , то дивизор $K_{X}+D$ называется $n$-полудополняемым, если сушествует $\mathbb{Q}$-дивизор $D^{+}$, удовлетворяюший требованиям 1), 3) и следуюшему условию:

$2^{\prime}$ ) дивизор $K_{X}+D^{+}$полулогканоничен. 
ПРЕДЛОЖЕНИЕ 1.7 [1; пример 5.2], [10; теорема 19.4]. Пусть X - полугладкая связная полная кривая. Пусть граница $D$ содержится в гладкой части $X$. Предположим, что дивизор $-\left(K_{X}+D\right)$ численно әффективен на каждой компоненте $X$. Тогда

1) дивизор $K_{X}+D \quad n$-полудополняем для $n \in\{1,2,3,4,6\}$;

2) если дивизор $K_{X}+D$ не 1- и 2-полудополняем, то $X \cong \mathbb{P}^{1} u\lfloor D\rfloor=$ $\left\lfloor D^{+}\right\rfloor=0$.

Инвариантность дополнений относительно логпрограммы минимальных моделей и их индуктивные свойства показаны в следующих утверждениях.

ПредлОЖенИЕ 1.8 [1; лемма 5.4]. Пусть $f: X \rightarrow Z$ - бирациональное стягивание многообразий и дивизор $D$ - субграница на $X$. Если дивизор $K_{X}+D$ является $n$-дополняемым, то дивизор $K_{Z}+f(D)$ тоже $n$-дополняем.

ПреДЛОЖЕнИЕ 1.9 [2; лемма 4.4]. Пусть $f: X \rightarrow Z$ - бирациональное стягивание многообразий и дивизор $D$ - субграница на X. Предположим, что

1) дивизор $K_{X}+D \quad f$-численно әффективен;

2) коэффичиент любой неисключительной, неприводимой компоненты дивизора $D$, пересекающей $\operatorname{Exc} f$, лежит в $\mathscr{P}_{n}$;

3) дивизор $K_{Z}+f(D)$ n-дополняем.

Тогда дивизор $K_{X}+D$ тоже $n$-дополняем.

Следующая теорема для двумерных многообразий усиливает обычную теорему о продолжении дополнений для $n$-мерных многообразий [3; предложение 4.4.1].

TЕорема 1.10 [3; предложение 4.4.3]. Пусть $(X / Z \ni P, D=S+B)$ - логnоверхность со следующими свойствами:

1) дивизор $K_{X}+D$ дивизориально логтерминален;

2) дивизор $-\left(K_{X}+D\right)$ численно эффективен и обгемен над $Z$;

3) $S=\lfloor D\rfloor \neq 0$ в окрестности $f^{-1}(P)$.

Предположим, что в окрестности $f^{-1}(P) \cap S$ существует п-полудополнение $K_{S}+\operatorname{Diff}_{S}(B)^{+}$для $K_{S}+\operatorname{Diff}_{S}(B)$. Тогда в окрестности $f^{-1}(P)$ существует $n$-дополнение $K_{X}+S+B^{+}$для $K_{X}+S+B$ mакое, ито $\operatorname{Diff}_{S}(B)^{+}=\operatorname{Diff}_{S}\left(B^{+}\right)$.

ОПРЕДЕЛЕНИЕ 1.11. Пусть $(X / Z \ni P, D)$ - стягивание многообразий. Если $\operatorname{dim} Z \neq 0$, то стягивание называется исключительныц, если для любого $\mathbb{Q}$-дополнения $D^{\prime}$ сушествует не более одного дивизора $E$ (не обязательно исключительного) такого, что $a\left(E, D^{\prime}\right)=-1$. Если $\operatorname{dim} Z=0$, то логмногообразие называется исключительныц, если для любого $\mathbb{Q}$-дополнения $D^{\prime}$ пара $\left(X, D^{\prime}\right)$ логтерминальна по Кавамате.

ОПРЕДЕЛЕниЕ 1.12. Пусть $(X, D)$ - исключительная пара. Положим

$$
\begin{array}{r}
\delta(X, D)=\#\{E \mid E-\text { дивизор (не обязательно исключительньй }) \\
\text { с дискрепантностью } \left.a(E, D) \leqslant-\frac{6}{7}\right\} .
\end{array}
$$


Лемма 1.13. Пусть $(X \ni P, \alpha C+B)$ является ростком двумерной логтерминальной парьи, где $(X \ni P)$ - нечиклическая особая точка, $C$ - кривая, $B \geqslant 0 u \alpha \geqslant 0$. Тогда

1) дивизор $K_{X}+\alpha C+B$ не является $(1-\alpha)$-логтерминальным;

2) дивизор $K_{X}+\alpha C+B$ является строго $(1-\alpha)$-логканоническим только в случае $(X \ni P, \alpha C+B) \cong$ an $\left(\mathbb{C}^{2} \ni 0, \alpha\{x y=0\}\right) / \mathbb{D}_{n}$, әде $\mathbb{D}_{n} \subset \mathrm{SL}_{2}(\mathbb{C})$ - диәдральная подгруппа.

ДокаЗАТЕльство. Для некоторого числа $0<c \leqslant 1$ пара $(X, c C+B)$ будет логканоничной, но не чисто логтерминальной [3; теорема 2.1.2]. Пусть $f:(Y, E) \rightarrow$ $(X \ni P)$ - индуктивное раздутие этой пары [11; теорема 1.9], [3; предложение 3.1.4]. Тогда $K_{Y}-f$-численно эффективньй дивизор, так как $f$ является раздутием центральной вершины графа минимального разрешения $[3 ; \S 6]$. Поэтому

$$
a(E, \alpha C+B)=-\frac{\alpha}{c}+\left(1-\frac{\alpha}{c}\right) a(E, B) \leqslant-\frac{\alpha}{c} \leqslant-\alpha .
$$

Равенство достигается только в случае $c=1, B=0, a(E, 0)=0$. Из классификации двумерных логтерминальных особенностей получаем требуемое (см., например, [3; теорема 2.1.2]).

Лемма 1.14. Пусть ( $X \ni P, \alpha C+B)$ является ростком двумерной логтерминальной пары, где $(X \ni P)$ - ииклическая особая точка, $C$ - кривая, $B=\sum b_{i} B_{i} \geqslant 0 u \alpha \geqslant 0$. Предположим, что пара $(X, C+B)$ не чисто логтерминальна и $b_{i} \geqslant \frac{1}{2}$ для всех $i$. Тогда

1) дивизор $K_{X}+\alpha C+B$ не является $(1-\alpha)$-логтерминальным ;

2) дивизор $K_{X}+\alpha C+B$ является строго $(1-\alpha)$-логканоническим только в следующих случаях:

a) $(X \ni P, \alpha C+B) \cong$ an $\left(\mathbb{C}^{2} \ni 0, \alpha\{x y=0\}\right) / \mathbb{Z}_{n}(n-1,1)$;

b) $(X \ni P, \alpha C+B) \cong$ an $\left(\mathbb{C}^{2} \ni 0, \alpha\left\{x^{2}+y^{2}=0\right\}\right) / \mathbb{Z}_{4}(3,1)$;

c) $(X \ni P, \alpha C+B) \cong$ an $\left(\mathbb{C}^{2} \ni 0, \alpha\left\{x^{2}+y^{4}=0\right\}\right) / \mathbb{Z}_{2}(1,1)$, əде $\alpha \leqslant \frac{2}{3}$;

d) $(X \ni P, \alpha C+B) \cong$ an $\left(\mathbb{C}^{2} \ni 0, \frac{1}{2}\{x=0\}+\frac{1}{2}\left\{x+y^{3}=0\right\}\right) / \mathbb{Z}_{2}(1,1)$.

ДокАЗАТЕЛЬСтво. Предположим, что $C$ является приводимой кривой или $B$ состоит не менее чем из двух дивизоров. Пусть $\psi: \widetilde{X} \rightarrow X-$ минимальное разрешение и $\Gamma$ - его граф. Собственные прообразы $C$ и $B$ обозначим через $\widetilde{C}$ и $\widetilde{B}$. Кривую из $\Gamma$, которую пересекает $\widetilde{C}$, обозначим через $\widetilde{E}$ и стянем все другие кривые из Г. Получили раздутие $f:(Y, E) \rightarrow(X \ni P)$. Запишем

$$
K_{Y}+a E+B_{Y}+C_{Y}=f^{*}\left(K_{X}+C+B\right) .
$$

Поскольку

$$
0 \leqslant-2+\operatorname{deg} \operatorname{Diff}_{E}(0)+\left(B_{Y}+C_{Y}\right) E=(1-a) E^{2},
$$

то $a \geqslant 1$. Следовательно,

$$
a(E, \alpha C+B)=-\alpha a+(1-\alpha) a(E, B) \leqslant-\alpha a \leqslant-\alpha .
$$

Равенство достигается только в случае $a=1, B=0, a(E, 0)=0$. Из классификации двумерных логтерминальных особенностей получаем подслучай а) или подслучай d), разобранный ниже (при этом $B=0$ ). 
Предположим, что $C$ является неприводимой кривой и $B$ состоит не более чем из одного дивизора. Если $(X \ni P) \varlimsup_{\text {an }}\left(\mathbb{C}^{2} \ni 0\right) / \mathbb{Z}_{n}(1,1)$, то, как и выше, можем найти кривую $E$, для которой выполнено неравенство (1). В случае равенства в $(2)$ получаем подслучай b).

Пусть $(X \ni P) \cong$ an $\left(\mathbb{C}^{2} \ni 0\right) / \mathbb{Z}_{n}(1,1)$. Положим $E=\operatorname{Exc} \psi$. При $(\widetilde{C}+\widetilde{B}) \cdot E \geqslant 2$ лемма доказьвается аналогично предыдущим случаям. В случае равенства в $(2)$ получаем подслучай с). Поэтому будем считать, что

$$
(X \ni P, C) \cong \text { an }\left(\mathbb{C}^{2} \ni 0,\{x=0\}\right) / \mathbb{Z}_{n}(1,1), \quad B=b_{1} B_{1}, \quad \widetilde{C} \cap \widetilde{B}_{1} \cap E=P .
$$

Раздуем (обыкновенньм образом) точку $P$. Тогда

$$
a\left(E^{\prime}, \alpha C+B\right)=-\alpha\left(1+\frac{1}{n}\right)-b_{1}\left(1+\frac{1}{n}\right)+\frac{2}{n},
$$

где $E^{\prime}$ - появившаяся исключительная кривая. Легко проверить, что неравенство $a\left(E^{\prime}, \alpha C+B\right)>-\alpha$ выполняется только в случае $n=2$ и $\alpha<\frac{1}{2}$, но тогда $a(E, \alpha C+B) \leqslant-\frac{\alpha}{2}-\frac{1}{4}<-\alpha$. Более того, $a\left(E^{\prime}, \alpha C+B\right)=-\alpha$ только в случае $n=2, \alpha=b_{1}=\frac{1}{2}$, т.е. имеем подслучай $\mathrm{d}$ ) (при этом $B \neq 0$ ).

ПРЕДЛОЖЕНИЕ 1.15. Пусть nара $(X \ni P, \alpha C+B)$ имеeт mun $\Phi_{i}$, где $i=2,3,4,5,6$ и $\alpha \geqslant(i-1) / i$. Тогда она является одной из следующих:

1) $(X \ni P, \alpha C+B) \cong$ an $\left(\mathbb{C}^{2} \ni 0, \alpha\{x=0\}+\beta\left\{x+y^{k}=0\right\}\right)$, zде $i=2, k \geqslant 2$, $\alpha>\frac{1}{2}, \beta>\frac{1}{2}, \alpha+\beta<1+\frac{1}{2 k}$

2) $(X \ni P, \alpha C+B) \cong$ an $\left(\mathbb{C}^{2} \ni 0, \alpha\{x=0\}+\frac{1}{2}\left\{x+y^{k}=0\right\}\right)$, zде $k \geqslant 2$; если $k=2$, то $i$ любое; если $k=3$, то $i=2,3$; если $k \geqslant 4$, то $i=2 u$ $\alpha<\frac{1}{2}+\frac{1}{2 k}$

3) $(X \ni P, \alpha C+B) \cong$ an $\left(\mathbb{C}^{2} \ni 0, \alpha\left\{x^{2}+y^{k}=0\right\}\right)$, әде $k \geqslant 2 ;$ ecли $k=2$, mо $i$ любое; если $k=3$, то $i=2,3,4$; если $k \geqslant 4$, то $i=2$ u $\alpha<\frac{1}{2}+\frac{1}{4[k / 2]}$;

4) $(X \ni P, \alpha C+B) \cong$ an $\left(\mathbb{C}^{2} \ni 0, \alpha\{x=0\}+b_{1}\{y=0\}\right) / \mathbb{Z}_{n}(q, 1)$, əдe $(n, q)=1 u\left(n / i-1+b_{1}\right) /(1-\alpha)<q \leqslant n$ (возможсен случай $\left.n=q=1\right)$.

ДокАЗАТЕльство. По лемме $1.13(X \ni P)$ является циклической особенностью или неособой точкой. Условие $\frac{1}{i}$-логтерминальности и вид коэффициентов дивизора $D$ являются основньми в доказательстве предложения.

Предположим, что дивизор $K_{X}+C+B$ чисто логтерминален. Если $(X \ni P)$ - неособая точка, то $(X \ni P, \alpha C+B) \cong$ an $\left(\mathbb{C}^{2} \ni 0, \alpha\{x=0\}+b_{1}\{y=0\}\right)$. Поступая таким же образом, как и при доказательстве предложения 1.9 [9], получаем случай 4).

Предположим, что дивизор $K_{X}+C+B$ не чисто логтерминален. Тогда по лемме $1.14(X \ni P)$ - неособая точка. Если дивизор $K_{X}+C+B$ является дивизориально логтерминальным, то имеем случай 3 ) с $k=2$. Предположим, что он не дивизориально логтерминален. Тогда имеются две возможности для дивизора $B$.

Пусть $B=b_{1} B_{1} \neq 0$. Тогда $C$ - неособая кривая и $B_{1}$ касается $C$ в точке $P$. Поэтому имеем случаи 1) и 2 ).

Пусть $B=0$. Тогда $C$ - особая кривая и имеем случай 3$)$ с $k \geqslant 3$.

ЗАмечание 1.16 . Случай $\Phi_{\mathrm{m}}=\bigcup_{i \geqslant 7}^{\infty} \Phi_{i}$ разобран в [9; предложение 1.9]. 


\section{§2. Основная индуктивная теорема для поверхностей}

Следуюшая теорема 2.1 уточняет основную индуктивную теорему о дополнениях на поверхностях [2; теорема 2.3].

ТЕОРема 2.1. Пусть $\left(S, D=\sum d_{i} D_{i}\right)$ - проективная логповерхность. Предполохсим, что выполнень следующие условия:

1) дивизор $K_{S}+D$ логканоничен, но не логтерминален по Кавамате;

2) $-\left(K_{S}+D\right)$ - численно әффективный дивизор;

3) существует $\mathbb{Q}$-дополнение для $K_{S}+D$;

4) $d_{i} \geqslant \frac{1}{2} \partial$ дя всех $i$.

Тогда для $K_{S}+D$ существует 1-, 2-, 3-, 4- или 6-нелогтерминальное по Кавамате дополнение, кроме случаев из примера 2.3.

Кроме того, если имеется бесконечно много дивизоров Е с дискрепантностьюа $(E, D)=-1$, то для $K_{S}+D$ существует 1- или 2-нелогтерминальное по Кавамате дополнение.

ДокАЗАТЕльство. Настоящая теорема во многих случаях верна без требования 4). При ее доказательстве будем следовать работе [2]. Случаи, где используется требование 4), будут полностью разобраны.

Рассматривая минимальную логтерминальную модификацию [3; определение 3.1.3], можно считать, что $(S, D)$ является дивизориально логтерминальной парой. Положим $C=\lfloor D\rfloor \neq 0$ и $B=\{D\}$. В зависимости от численной размерности дивизора $-\left(K_{S}+C+B\right)$ имеем три случая.

Случай І. Предположим, что $-\left(K_{S}+C+B\right)$ - объемньй дивизор. Тогда из предложения 1.7 и теоремы 1.10 немедленно следуют все требуемые утверждения. Заметим, что в этом случае требование 4) на границу $D$ является излишним.

Перед тем как перейти к оставшимся двум случаям, уточним строение логповерхности $(S, D)$.

Пусть $S$ - нерациональная поверхность. Тогда наша теорема доказана в [2; теорема 2.3], [3; теорема 8.2.1]. Более того, всегда сушествует 1- или 2-нелогтерминальное по Кавамате дополнение и имеется лишь конечное число (не более двух) дивизоров $E$ с дискрепантностью $a(E, D)=-1$. Заметим, что и в этом случае требование 4 ) на границу $D$ является излишним.

Пусть $C$ не является цепю рациональных кривых. Тогда наша теорема также верна вне зависимости от требования 4) на коэффициенты границы $D[2$; теоремa 2.3].

Итак, будем считать, что $S$ - рациональная поверхность и $C$ - цепь рациональных кривых.

Случай II. Предположим, что $K_{S}+C+B \not \equiv 0$ и $-\left(K_{S}+C+B\right)$ не является объемным дивизором. По предложению 2.5 [2] можно считать, что дивизор $-\left(K_{S}+C+B\right)$ полуобилен. Пусть $\nu: S \rightarrow Z \cong \mathbb{P}^{1}$ - морфизм, заданный линейной системой $\left|-m\left(K_{S}+C+B\right)\right|$ для $m \gg 0$. Следуюшая лемма является основной при построении дополнений.

ЛЕмма 2.2 [2; леммы 2.20, 2.21]. Пусть $(S, D)$ - проективная логповерхность со структурой расслоения на кривую $f: S \rightarrow Z$, где $D$ - граница. Пусть 
$C=\lfloor D\rfloor \neq 0$ и $B=\{D\}$. Предположим, что выполнены следующие условия:

1) существует сечение $C_{1} \subset C$ для $f$;

2) дивизор $K_{C}+\operatorname{Diff}_{C}(B)$ n-полудополняем;

3) дивизор $K_{S}+C+\lfloor(n+1) B\rfloor / n$ численно тривиален на общем слое;

4) дивизор $-\left(K_{S}+C+B\right)$ численно әффективен;

5) дивизор $K_{S}+C+B$ логтерминален в (аналитической) окрестности дивизора $C$.

Тогда дивизор $K_{S}+C+B$ имеет п-дополнение. Более того, условия 1) и 3) можно заменить на условие $\left.1^{\prime}\right)$ :

$\left.1^{\prime}\right)$ существует мультисечение $C_{1} \subset C$ для $f$ и $S$-рачиональная поверхность.

Имеем три возможности для $C$.

A) Пусть $C_{1} \subset C$ - это мультисечение для $\nu$. Тогда вне зависимости от условия 4$)$ на $D$ по лемме 2.2 и предложению 1.7 имеем требуемые утверждения.

В) Пусть $C$ имеет единственное сечение $C_{1}$ для $\nu$. Лемма 2.2 не может быть применена только в случае, если в $B$ найдется горизонтальная компонента $B_{i}$ с коэффицциентом $b_{i} \in \mathbb{Z} /(n+1)$ [2; лемма 2.27$]$.

$\mathrm{C}$ другой стороны, если в $B$ найдется горизонтальная компонента $B_{j}$ с коэффициентом $b_{j} \notin \mathbb{Z} /(n+1)$, то рассмотрим дивизор $K_{S}+C+B-\varepsilon B_{j}$ при $0<\varepsilon \ll 1$. Он имеет такие же $n$-дополнения, как и дивизор $K_{S}+C+B$ (см. определение 1.6). Поскольку $-\left(K_{S}+C+B\right)+\varepsilon B_{j}$ является численно эффективным и объемным дивизором, то теорема сведена к случаю І. Поэтому предполагаем, что все горизонтальные компоненты $B$ имеют коэффициенты из множества $\mathbb{Z} /(n+1)$.

Предположим, что $C$ - приводимый дивизор. Тогда по предложению 1.7 дивизор $K_{C}+\operatorname{Diff}_{C}(B) 1$ - или 2-полудополняем. Если он 1-полудополняем, то $B^{\text {hor }}=$ $\frac{1}{2} B_{1}+\frac{1}{2} B_{2}$ или $B^{\text {hor }}=\frac{1}{2} B_{1}$ и, следовательно, имеем 2 -дополнение для $K_{S}+C+B$. Если он 2-полудополняем, то имеем противоречие с условием 4$)$.

Предположим, что $C=C_{1}$. Тогда из условия 4) имеем $n=1,3$. При $n=1$, как и вьше, сушествует 2-дополнение для $K_{S}+C+B$. Рассмотрим случай $n=3$. Тогда $B^{\text {hor }}=\frac{1}{2} B_{1}+\frac{1}{2} B_{2}$ или $B^{\text {hor }}=\frac{1}{2} B_{1}$. Дивизор $K_{S}+C+B$ не имеет 1-, 2-, 3-, 4 - и 6-дополнения только в случае, когда дивизор $K_{C}+\operatorname{Diff}_{C}(B)$ не имеет 1-,2-,4и 6 -дополнения, т.е. (после несложных вычислений)

$$
\left(C, \operatorname{Diff}_{C}(B)\right)=\left(\mathbb{P}^{1},\left(\frac{3}{5}+\varepsilon_{1}\right) P_{1}+\left(\frac{2}{3}+\varepsilon_{2}\right) P_{2}+\left(\frac{5}{7}+\varepsilon_{3}\right) P_{3}\right),
$$

где $\varepsilon_{1}+\varepsilon_{2}+\varepsilon_{3}<\frac{2}{105}$ и $\varepsilon_{i} \geqslant 0$ для всех $i$. По лемме 2.2 дивизор $K_{S}+C+B$ имеет 12 -дополнение (не всегда минимального индекса).

Пусть $\nu: S \stackrel{\psi_{1}}{\longrightarrow} S^{\prime} \longrightarrow Z-$ стягивание всех кривых в слоях $\nu$ (с помощью ЛПММ) таких, что $\left(K_{S}+C+\lfloor 13 B\rfloor / 12\right) \cdot E>0$. Так как $\left(K_{S}+C+\right.$ $\lfloor 13 B\rfloor / 12) \cdot C=0$, то $\psi_{1}$ не стягивает кривые, пересекающие $C$. Получили, что дивизор $\left(K_{S^{\prime}}+C^{\prime}+\left\lfloor 13 B^{\prime}\right\rfloor / 12\right)$ численно эффективен и, в частности, численно тривиален над $Z$, где $C^{\prime}$ и $B^{\prime}$ - образы $C$ и $B$. Конус $\overline{\mathrm{NE}}(S / Z)$ является полиэдральным и порождается стягиваемыми экстремальными кривыми $[2$; предложение 2.5]. Пусть $\nu: S \stackrel{\psi_{1}}{\longrightarrow} S^{\prime} \stackrel{\psi_{2}}{\longrightarrow} \bar{S} \longrightarrow Z$ - стягивание всех кривых в слоях $\nu$, 
непересекающих $C^{\prime}$. Тогда $\rho(\bar{S} / Z)=1$. Пара $\left(\bar{C}, \operatorname{Diff}_{\bar{C}}(\bar{B})\right)$ является такой же, как и в $(*)$, где $\bar{C}$ и $\bar{B}$ - образы $C^{\prime}$ и $B^{\prime}$. Заметим, что либо $\operatorname{Diff} \bar{C}(0)=0$, либо $\operatorname{Diff}_{\bar{C}}(0)=\frac{2}{3} P_{2}$ и $\varepsilon_{2}=0$.

i) Рассмотрим случай $\operatorname{Diff}_{\bar{C}}(0)=0$. Так как $\bar{C} \bar{B}^{\text {hor }}=0$, то $\bar{C}^{2} \leqslant 0$. Следовательно, при $m \gg 0$ линейная система $|\bar{C}+m f|$ задает бирациональный морфизм $\psi: \bar{S} \rightarrow \mathbb{F}_{k}$, где $f$ - общий слой [9; предложение 1.10$]$. Получили $\bar{S} \cong \mathbb{F}_{k}$.

ii) Рассмотрим случай Diff $\bar{C}_{\bar{C}}(0)=\frac{2}{3} P_{2}$ и $\varepsilon_{2}=0$. Пусть $\phi: \bar{S}^{\prime} \rightarrow \bar{S}$ - раздутие с единственной исключительной кривой в точке $P_{2}$, после которого Sing $\bar{S}^{\prime} \cap \bar{C}^{\prime}=\varnothing$ и дивизор $K_{\bar{S}^{\prime}} \phi$-численно эффективен. Положим $f_{2}=\operatorname{Exc} \phi$. Аналогично предыдушему случаю, линейная система $\left|\bar{C}^{\prime}+m f\right|$ задает бирациональный морфизм $\psi: \bar{S}^{\prime} \rightarrow \mathbb{F}_{k}$. Пусть $E=\operatorname{Exc} \psi, \widehat{D}=\psi\left(\bar{C}^{\prime}\right)+\left\lfloor 13 \psi\left(\bar{B}^{\prime}\right)\right\rfloor / 12$ и $\psi(E)=P$. Тогда либо

$$
\left(\mathbb{F}_{k} \ni P, \widehat{D}\right) \cong \text { an }\left(\mathbb{C}_{x, y}^{2} \ni 0, \frac{2}{3}\{y=0\}+\frac{1}{2}\left\{x\left(x+y^{t}\right)=0\right\}\right),
$$

либо

$$
\left(\mathbb{F}_{k} \ni P, \widehat{D}\right) \cong \text { an }\left(\mathbb{C}_{x, y}^{2} \ni 0, \frac{2}{3}\{y=0\}+\frac{1}{2}\left\{x^{2}+y^{t}=0\right\}\right)
$$

Так как $a(E, \widehat{D})=0$ и $f_{2}^{2}<-1$, то $\psi$ является взвешенным раздутием с весами $(1,3)$ или $(2,3)$ (cp. [9; лемма 5.5]). При раздутии с весами $(2,3)$ во втором случае должно выполняться условие $t \geqslant 2$. В следуюшем примере подведен итог вышесказанному.

ПримеР 2.3. а) Пусть

$$
\left(\mathbb{F}_{k}, D^{+}\right)=\left(\mathbb{F}_{k}, E_{\infty}+\frac{1}{2} E_{1}+\frac{1}{2} E_{2}+\frac{7}{12} f_{1}+\frac{2}{3} f_{2}+\frac{3}{4} f_{3}\right),
$$

где $E_{\infty}$ - минимальное сечение, $E_{i}$ - нулевое сечение, $f_{i}$ - слой. Пусть $h: S \rightarrow \mathbb{F}_{k}$ - бирациональное стягивание:

$$
K_{S}+\widetilde{E}_{\infty}+\frac{1}{2} \widetilde{E}_{1}+\frac{1}{2} \widetilde{E}_{2}+\frac{7}{12} \widetilde{f}_{1}+\frac{2}{3} \widetilde{f}_{2}+\frac{3}{4} \widetilde{f}_{3}+\sum a_{i} \Delta_{i}=h^{*}\left(K_{\mathbb{F}_{k}}+D^{+}\right) \equiv 0
$$

Предположим, что $a_{i} \in\{0\} \cup\left[\frac{1}{2}, 1\right) \cup \mathbb{Z} / 12, h\left(\Delta_{i}\right) \notin E_{\infty}$ для всех $i$. Поскольку пара $\left(\mathbb{F}_{k}, D^{+}\right)$логтерминальна по Кавамате вне $E_{\infty}$, то по лемме 3.1 .9 [3] таких поверхностей $S$ конечное число. Логповерхность

$$
\begin{aligned}
(S, D)= & \left(S, \widetilde{E}_{\infty}+\frac{1}{2} \widetilde{E}_{1}+\frac{1}{2} \widetilde{E}_{2}+\left(\frac{3}{5}+\varepsilon_{1}\right) \widetilde{f}_{1}+\left(\frac{2}{3}+\varepsilon_{2}\right) \widetilde{f}_{2}\right. \\
& \left.+\left(\frac{5}{7}+\varepsilon_{3}\right) \widetilde{f}_{3}+\sum_{i: a_{i} \geqslant 1 / 2} a_{i} \Delta_{i}\right)
\end{aligned}
$$

удовлетворяет требованиям теоремы 2.1 , где $\varepsilon_{1}+\varepsilon_{2}+\varepsilon_{3} \leqslant \frac{2}{105}$ и $\varepsilon_{i} \geqslant 0$ для всех $i$. При $\frac{5}{7}+\varepsilon_{3}<\frac{8}{11}$ имеем 10-дополнение для $K_{S}+D$, а при $\frac{5}{7}+\varepsilon_{3} \geqslant \frac{8}{11}$ имеем 12 -дополнение. Следует отметить, что вполне возможно небольшое изменение коэффициентов $a_{i}$ и при $k>0$ можно стянуть $\widetilde{E}_{\infty}$. 


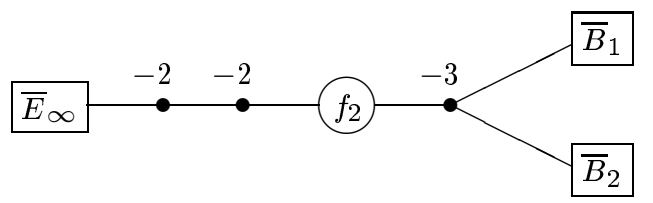

Рис. 1

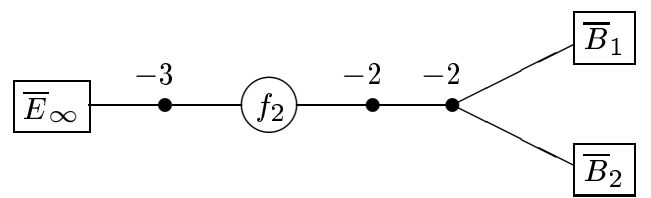

Рис. 2

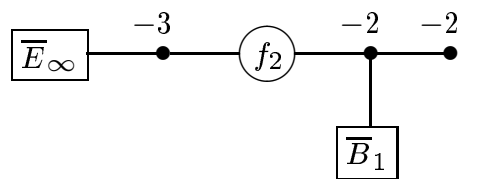

Рис. 3

b) Пусть $\bar{S} \rightarrow \mathbb{P}^{1}$ - экстремальное $\mathbb{P}^{1}$-расслоение (в обшей точке), т.е.

$$
\rho\left(\bar{S} / \mathbb{P}^{1}\right)=1
$$

Предположим, что Sing $\bar{S} \subset f_{2}$, где слой $f_{2}$ изображен на одном из рис. $1-3$.

Если рассмотреть минимальное разрешение поверхности $\bar{S}$, а затем стянуть все $(-1)$ кривые, не пересекающие собственный прообраз $\bar{E}_{\infty}$, то получим $\mathbb{F}_{k}$. На линейчатой поверхности $\mathbb{F}_{k}$ образ $\bar{E}_{\infty}$ будет минимальным сечением, образы $\bar{B}_{1}$ и $\bar{B}_{2}$ с рис. 1,2 будут сечениями, а образ $\bar{B}_{1}$ с рис. 3 будет 2 -мультисечением.

Рассмотрим логповерхность

$$
\left(\bar{S}, D^{+}\right)=\left(\bar{S}, \bar{E}_{\infty}+\bar{B}+\frac{7}{12} f_{1}+\frac{3}{4} f_{3}\right)
$$

где $\bar{B}=\frac{1}{2} \bar{B}_{1}+\frac{1}{2} \bar{B}_{2}$ (в случае рис. 1 или 2 ) или $\bar{B}=\frac{1}{2} \bar{B}_{1}$ (в случае рис. 3 ). Для нее аналогично п. 1) строятся бирациональные морфизмы $h: S \rightarrow \bar{S}$. Очевидно, что имеют место те же самые утверждения о строении $h$ и о дополняемости $K_{S}+D$.

С) Пусть $C$ лежит в слое $\nu$. Положим $P=\nu(C)$ и $f=\nu^{-1}(P)$. Случай, когда обший слой - эллиптическая кривая, рассмотрен в III.В). Поэтому считаем, что общий слой $\nu$ является рациональной кривой. Дивизор $K_{C}+\operatorname{Diff}_{C}(B)$ имеет $n$-полудополнение минимального индекса, где $n \in\{1,2,3,4,6\}$ (см. предложение 1.7 ). 
Предположим, что существует горизонтальная компонента $B_{i}$ с коэффициентом $b_{i} \notin \mathbb{Z} /(n+1)$. Тогда, рассматривая дивизор $K_{S}+C+B-\varepsilon B_{i}$, сводим задачу к случаю I. Поэтому предполагаем, что все горизонтальные компоненты $B$ имеют коэффициенты из множества $\mathbb{Z} /(n+1)$. Покажем, что это невозможно.

Пусть $\nu: S \stackrel{\psi}{\longrightarrow} S^{\prime} \longrightarrow Z$ - стягивание всех кривых в слое $f$, не лежащих в $C$. Положим $C^{\prime}=\psi(C), B^{\prime}=\psi(B)$. Так как $K_{S}+C+B \equiv 0$ над $Z$, то $\left(C, \operatorname{Diff}_{C}(B)\right)=$ $\left(C^{\prime}, \operatorname{Diff}_{C^{\prime}}\left(B^{\prime}\right)\right)$.

Предположим, что $C$ - приводимая кривая. Тогда $n=1,2$. Если $n=1$, то по условию 4 ) коэффициенты всех горизонтальных компонент $B$ равны $\frac{1}{2}$. Поскольку дивизор $K_{S^{\prime}}+C^{\prime}+B^{\prime}$ дивизориально логтерминален и численно тривиален над $Z$, то в $\operatorname{Diff}_{C^{\prime}}\left(B^{\prime}\right)$ существует дивизор с коэффициентом $\frac{1}{2}$. Противоречие с $n=1$. Если $n=2$, то имеем аналогичное противоречие.

Случай, когда $C$ является неприводимой кривой, рассматривается таким же образом.

Случай III. Предположим, что $K_{S}+C+B \equiv 0$.

ОПРЕДЕЛЕНИЕ 2.4. Пусть $D$ является $\mathbb{Q}$-дивизором на проективном многообразии $X$. Определим численную размерность дивизора $D$ :

$$
\nu_{\text {num }}(D)=\max \left\{\nu_{\text {num }}\left(D^{\prime}\right) \mid D^{\prime} \geqslant 0, \operatorname{Supp} D^{\prime} \subseteq \operatorname{Supp} D\right\} .
$$

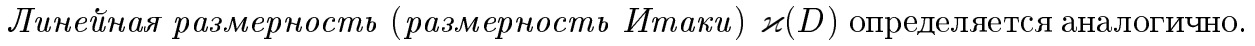

Имеем $\varkappa(B)=\nu_{\text {num }}(B)[2 ;$ предложение 2.12]. Рассмотрим по порядку все возможности для $\nu_{\text {num }}(B)$.

А) Пусть $\nu_{\text {num }}(B)=2$. Конус $\overline{\mathrm{NE}}(S)$ является полиэдральным и порождается стягиваемыми экстремальными кривьми, так как сушествует дивизор $B^{\prime}$ $\left(\operatorname{Supp} B^{\prime} \subseteq \operatorname{Supp} B\right)$, для которого дивизор $-\left(K_{S}+C+B\right)+\varepsilon B^{\prime}$ является численно эффективным и объемным [2; предложение 2.5]. Пусть дивизор $K_{C}+$ $\operatorname{Diff}_{C}(B) n$-полудополняем. Стянем все исключительные кривые $E$ при условии $\left(K_{S}+C+\lfloor(n+1) B\rfloor / n\right) \cdot E>0$. В итоге получим $\nu: S \stackrel{\psi}{\longrightarrow} S^{\prime} \stackrel{\nu^{\prime}}{\longrightarrow} Z \cong \mathbb{P}^{1}$ и $\left(K_{S^{\prime}}+C^{\prime}+\left\lfloor(n+1) B^{\prime}\right\rfloor / n\right) \cdot f>0$, где $f$ - обший слой $\nu^{\prime}$, либо $\psi: S \rightarrow S^{\prime}$ и $-\left(K_{S^{\prime}}+C^{\prime}+\left\lfloor(n+1) B^{\prime}\right\rfloor / n\right)$ - численно эффективньй дивизор. По построению никакая из компонент $C$ не стягивается $\psi$ и в первом случае $C^{\prime}$ не лежит в слоях $\nu^{\prime}$. Поэтому в первом случае $C$ имеет горизонтальные компоненты для $\nu$. Более того, так как $\nu_{\text {num }}(B)=2$, то по условию 4 ) имеем $B^{\text {hor }}=\frac{1}{2} B_{1}+\frac{1}{2} B_{2}$ или $B^{\text {hor }}=\frac{1}{2} B_{1}$. Таким образом, первый случай сведен к случаю II.В). В пример 2.3 была включена эта возможность.

Во втором случае вне зависимости от коэффициентов границы $B$ доказано, что дивизор $K_{S^{\prime}}+C^{\prime}+\left\lfloor(n+1) B^{\prime}\right\rfloor / n \quad n$-дополняем [2] (здесь сушественно, что конус $\overline{\mathrm{NE}}\left(S^{\prime}\right)$ является полиэдральным и порождается стягиваемыми экстремальньми кривыми). По предложению 1.9 дивизор $K_{S}+C+B n$-дополняем.

В) Пусть $\nu_{\text {num }}(B)=1$. Тогда для некоторого $B^{\prime}\left(\operatorname{Supp} B^{\prime} \subseteq \operatorname{Supp} B\right)$ линейная система $\left|B^{\prime}\right|$ задает расслоение $\nu: S \rightarrow Y$ и дивизор $B$ лежит в слоях. Если некоторая компонента $C$ является сечением, то общий слой - это $\mathbb{P}^{1}$. Следовательно, сушествует мультисечение $C_{1} \subset C$. По лемме 2.2 теорема доказана. Поэтому будем считать, что $C$ лежит в слоях и общий слой - это эллиптическая кривая. Поступая, как и ранњше, стянем кривые $E \not \subset \operatorname{Supp} C$ такие, 
что $\left(K_{S}+C+\lfloor(n+1) B\rfloor / n\right) E \geqslant 0$, где $n$ - индекс полудополняемости для $K_{S}+\operatorname{Diff}_{C}(B)$. В итоге получим $\nu: S \stackrel{\phi}{\longrightarrow} S^{\prime} \stackrel{\nu^{\prime}}{\longrightarrow} Y$, где все слои $\nu^{\prime}$ являются неприводимьми, за исключением слоя, состоящего из $C^{\prime}$. Пусть $\psi: S^{\prime} \rightarrow S^{\prime \prime}-$ стягивание компонент $C^{\prime}$. Получим модель с $\rho\left(S^{\prime \prime}\right)=2$. Конус поверхности $S^{\prime \prime}$ имеет два экстремальных луча: слой $\nu^{\prime}$, (мульти)сечение $E$. Если $\left(K_{S^{\prime \prime}}+C^{\prime \prime}+\right.$ $\left.\left\lfloor(n+1) B^{\prime \prime}\right\rfloor / n\right) \cdot E \leqslant 0$, то дивизор $-\left(K_{S^{\prime}}+C^{\prime}+\left\lfloor(n+1) B^{\prime}\right\rfloor / n\right)$ численно эффективен. В этом случае теорема доказана в [2] вне зависимости от коэффициентов $B$. Если $\left(K_{S^{\prime \prime}}+C^{\prime \prime}+\left\lfloor(n+1) B^{\prime \prime}\right\rfloor / n\right) \cdot E>0$, то имеем расслоение $\theta: S \rightarrow Z \cong \mathbb{P}^{1}$ и $C$ содержит сечение для $\theta$. Горизонтальная часть дивизора $B$ для $\theta$ по условию 4$)$ имеет вид $B^{\text {hor }}=\frac{1}{2} B_{1}+\frac{1}{2} B_{2}$ или $B^{\text {hor }}=\frac{1}{2} B_{1}$. Поскольку $K_{S}+C+B \equiv 0$ над $Y$ и $K_{S^{\prime \prime}}+C^{\prime \prime} \equiv 0$ над $Y$, то $\operatorname{Diff}_{C}(B)=\operatorname{Diff}_{C^{\prime \prime}}(0)$. Следовательно, коэффициенты $\operatorname{Diff}_{C}(B)$ являются стандартньми. В частности, при $n=1,3$ имеем $\operatorname{Diff}_{C}(B)=P_{1}+P_{2}$ и Diff $C(B)=\frac{2}{3} P_{1}+\frac{2}{3} P_{2}+\frac{2}{3} P_{3}$ соответственно. Поэтому по лемме 2.2 при $n=1,2,3,4,6$ дивизор $K_{S}+C+B$ имеет 2-, 2-, 6-, 4-, 6-дополнение соответственно.

C) Пусть $\nu_{\text {num }}(B)=0$, т.е. дивизор $B$ стягивается. Тогда вне зависимости от коэффициентов границы $D$ имеем требуемые утверждения [2].

ЗАмЕчАниЕ 2.5. Условие 3 ) теоремы 2.1 можно заменить на одно из более сильных следующих условий (см. [2; предложение 2.5]):

$\left.3^{1}\right)$ дивизор $-\left(K_{S}+D\right)$ объемен;

$\left.3^{2}\right)$ конус $\overline{\mathrm{NE}}(S)$ является полиэдральным и порождается стягиваемыми экстремальными кривыми;

$\left.3^{3}\right)$ дивизор - $\left(K_{S}+D\right)$ полуобилен;

$\left.3^{4}\right)$ сушествует стягивание $\nu: S \rightarrow Z$ такое, что если $(K+D) \cdot E=0$, то $E \subset \operatorname{Exc} \nu$.

В примере 2.3 логповерхности $(S, D)$ удовлетворяют всем перечисленным условиям, конечно, кроме условия $\left(3^{1}\right)$.

Следующее следствие является очень важным для приложений.

СлеДСТВИЕ 2.6. Пусть $\left(S, D=\sum d_{i} D_{i}\right)$ - проективная логповерхность. Предположим, что выполнень следующие условия:

1) дивизор $K_{S}+D$ логтерминален по Кавамате;

2) $-\left(K_{S}+D\right)$ - численно әффективный дивизор;

3) существует $\mathbb{Q}$-дополнение для $K_{S}+D$;

4) $d_{i} \geqslant \frac{1}{2} \partial$ ля всех $i$

5) существует эффективный $\mathbb{Q}$-дивизор $D^{\prime} \geqslant D$ такой, что $-\left(K_{S}+D^{\prime}\right)$ - численно әффективный дивизор и пара $\left(S, D^{\prime}\right)$ не логтерминальна по Кавамате.

Тогда для $K_{S}+D$ существует 1-, 2-, 3-, 4- или 6-нелогтерминальное по Кавамате дополнение, кроме случаев, возникающих в примере 2.3.

Кроме того, если имеется бесконечно много дивизоров Е с дискрепантностью а $\left(E, D^{\prime}\right)=-1$ и пара $\left(S, D^{\prime}\right)$ логканонична, то для $K_{S}+D$ существует 1- или 2-нелогтерминальное по Кавамате дополнение. 
ДокаЗАТЕЛЬСтво. Заменяя дивизор $D^{\prime}$ на $D+\lambda\left(D^{\prime}-D\right)$ для подходящего $\lambda>0$, будем считать, что дивизор $K_{S}+D^{\prime}$ логканоничен, но не логтерминален по Кавамате.

Сначала докажем, что для дивизора $K_{S}+D^{\prime}$ сушествует $\mathbb{Q}$-дополнение. Если дивизор $-\left(K_{S}+D\right)$ объемный, то конус $\overline{\mathrm{NE}}(S)$ является полиэдральным и порождается стягиваемьми экстремальными кривыми, и имеем требуемое $[2 ;$ предложение 2.5]. Поэтому можно считать, что линейная система $\left|-m\left(K_{S}+D\right)\right|$ для $m \gg 0$ задает расслоение $\nu: S \rightarrow Z$. Добавляя к $K_{S}+D^{\prime}$ нужное количество общих слоев морфизма $\nu$, имеем требуемое.

Пусть $f: \widetilde{S} \rightarrow S$ - минимальная логтерминальная модификация пары $\left(S, D^{\prime}\right)$ [3; определение 3.1.3]. Имеем

$$
K_{\widetilde{S}}+\sum E_{i}+\left\lfloor\widetilde{D}^{\prime}\right\rfloor+\left\{\widetilde{D}^{\prime}\right\}=f^{*}\left(K_{S}+D^{\prime}\right)
$$

где $\widetilde{D}^{\prime}$ - собственный прообраз $D^{\prime}$. Положим

$$
\widetilde{D}=\sum E_{i}+\left\lfloor\widetilde{D}^{\prime}\right\rfloor+\sum_{i: \operatorname{Supp}} \sum_{\widetilde{D}_{i} \not \subset \operatorname{Supp}\left\lfloor\widetilde{D}^{\prime}\right\rfloor} d_{i} \widetilde{D}_{i}
$$

где $\widetilde{D}_{i}-$ собственные прообразы $D_{i}$. Таким образом, утверждение следствия надо доказать для дивизора $K_{\widetilde{S}}+\widetilde{D}$. Если дивизор $-\left(K_{\widetilde{S}}+\widetilde{D}\right)$ численно эффективен, то по теореме 2.1 все доказано. Поэтому будем считать, что $-\left(K_{\widetilde{S}}+\widetilde{D}\right)-$ не численно эффективный дивизор. $\mathbb{Q}$-дополнение для дивизора $K_{\widetilde{S}}+\widetilde{D}$ обозначим через $\widetilde{\Theta}$. Можно считать, что $\lfloor\widetilde{\Theta}\rfloor=\lfloor\widetilde{D}\rfloor$.

Докажем, что можно последовательно стянуть все исключительные кривые $E$, для которых $\left(K_{\widetilde{S}}+\widetilde{D}\right) \cdot E>0$.

А) Предположим, что $\nu_{\text {num }}(\{\widetilde{\Theta}\})=2$. Тогда, как и в доказательстве теоремы 2.1 (случай III.А)), конус $\overline{\mathrm{NE}}(\widetilde{S})$ является полиэдральным и порождается стягиваемыми экстремальными кривыми, что и требовалось доказать.

В) Предположим, что $\nu_{\text {num }}(\{\widetilde{\Theta}\})=1$. По предложению 2.12 [2] для некоторого дивизора $\widetilde{\Theta}^{\prime}\left(\operatorname{Supp} \widetilde{\Theta}^{\prime} \subset \operatorname{Supp}\{\widetilde{\Theta}\}\right)$ линейная система $\left|\widetilde{\Theta}^{\prime}\right|$ задает расслоение $\nu: \widetilde{S} \rightarrow Z$ и дивизор $\{\widetilde{\Theta}\}$ лежит в слоях морфизма $\nu$. Если $\left(K_{\widetilde{S}}+\widetilde{D}\right) \cdot E>0$, то кривая $E$ лежит в слоях морфизма $\nu$. Поэтому ее можно стянуть.

С) Предположим, что $\nu_{\text {num }}(\{\widetilde{\Theta}\})=0$. Тогда по определению дивизор $\{\widetilde{\Theta}\}$ стягивается.

Итак, получили бирациональный морфизм $\phi: \widetilde{S} \rightarrow \bar{S}$. Ясно, что $\phi$ не стягивает компонент из $\lfloor\widetilde{D}\rfloor$, и на каждом шаге стягиваемая кривая пересекает некоторую компоненту $\widetilde{\Theta}_{1}$ из $\lfloor\widetilde{D}\rfloor$. Положим $\bar{D}=\phi(\widetilde{D})$.

Остается показать, что $n$-дополнение $\bar{D}^{+}$для $K_{\bar{S}}+\bar{D}$ поднимается до $n$-дополнения для $K_{\widetilde{S}}+\widetilde{D}(n=1,2,3,4$ или 6$)$. Положим

$$
K_{\widetilde{S}}+\widetilde{D}^{+}=\phi^{*}\left(K_{\bar{S}}+\bar{D}^{+}\right) .
$$

Надо доказать, что

$$
\lfloor\widetilde{D}\rfloor+\frac{\lfloor(n+1)\{\widetilde{D}\}\rfloor}{n} \leqslant\left\lfloor\widetilde{D}^{+}\right\rfloor+\left\{\widetilde{D}^{+}\right\} .
$$


По вышесказанному это требование достаточно проверить в случае, когда $\phi$ является стягиванием единственной кривой $E$. Из классификации двумерных логтерминальных пар [3; теорема 2.1.2] и условия 4) получаем, что через точку $P \in \bar{S}$, в которую стягивается $E$, проходит не более одного дивизора из $\{\bar{D}\}$, и эта точка является циклической факторособенностью. Пусть $d_{1} \bar{D}_{1}$ является дивизором, проходящим через $P$. Если коэффициент при дивизоре $\bar{D}_{1}$ в $\bar{D}^{+}$больше $d_{1}$, то мы рассматриваем его вместо $d_{1}$. Учитывая, что дивизор $K_{\lfloor\bar{D}\rfloor}+\operatorname{Diff}_{\lfloor\bar{D}\rfloor}(\{\bar{D}\}) n$-полудополняем, имеем следующие случаи (случай $n=1$ очевиден).

а) Если $\left(\bar{S} \ni P, \bar{\Theta}_{1}+d_{1} \bar{D}_{1}\right) \cong$ an $\left(\mathbb{C}^{2} \ni 0,\{x=0\}+d_{1}\{y=0\}\right) / \mathbb{Z}_{2}(1,1)$ и $n=4$, то по предложению 1.9 требование $(* *)$ надо проверить для $d_{1} \in\left(\frac{1}{2}, \frac{3}{5}\right)$.

b) Если $\left(\bar{S} \ni P, \bar{\Theta}_{1}+d_{1} \bar{D}_{1}\right) \cong$ an $\left(\mathbb{C}^{2} \ni 0,\{x=0\}+d_{1}\{y=0\}\right) / \mathbb{Z}_{2}(1,1)$ и $n=6$, то по предложению 1.9 требование $(* *)$ надо проверить для $d_{1} \in\left(\frac{2}{3}, \frac{5}{7}\right)$.

с) Если $\left(\bar{S} \ni P, \bar{\Theta}_{1}+d_{1} \bar{D}_{1}\right) \cong$ an $\left(\mathbb{C}^{2} \ni 0,\{x=0\}+d_{1}\{y=0\}\right) / \mathbb{Z}_{3}(q, 1)$ и $n=6$, то по предложению 1.9 требование $(* *)$ надо проверить для $d_{1} \in\left(\frac{1}{2}, \frac{4}{7}\right)$.

d) Если $\left(\bar{S} \ni P, \bar{\Theta}_{1}+d_{1} \bar{D}_{1}\right) \cong$ an $\left(\mathbb{C}^{2} \ni 0,\{x=0\}+d_{1}\{y=0\}\right)$, то требование $(* *)$ равносильно следуюшему:

$$
-\frac{\left\lfloor(n+1) a\left(E, \bar{\Theta}_{1}+d_{1} \bar{D}_{1}\right)\right\rfloor}{n} \leqslant-a\left(E, \bar{\Theta}_{1}+\frac{\left\lfloor(n+1) d_{1}\right\rfloor}{n} \bar{D}_{1}\right)
$$

Учитывая условие $a\left(E, \bar{\Theta}_{1}+d_{1} \bar{D}_{1}\right) \leqslant-\frac{1}{2}$, получаем, что $\phi$ является торическим раздутием. Требование (***) в случаях а)-c) проверяется непосредственно. В случае $\mathrm{d})$ веса взвешенного раздутия $\phi$ обозначим через $(\alpha, \beta)$. Тогда $(\alpha, \beta)=(\alpha, 1)$ и $d_{1} \geqslant \frac{1}{2}$, либо $(\alpha, \beta)=(\alpha, 2), d_{1} \geqslant \frac{3}{4}$ и $n=4$, либо $(\alpha, \beta)=(\alpha, 3), d_{1} \geqslant \frac{5}{6}$ и $n=6$. Теперь простые вычисления показывают, что требование $(* * *)$ также вьполняется.

СлЕДСТВИЕ 2.7. Если в формулировке следствия 2.6 отказаться от условия 4) на границу $D$, то для $K_{S}+D$ существует не логтерминальное по Кавамате п-дополнение, әде $n=1,2,3,4,5,6,7,8,9,10,11,12,13,14,15$, $16,17,18,19,20,21,22,23,24,25,26,27,28,29,30,31,35,36,40,41,42,43$, 56 или 57.

Кроме того, если имеется бесконечно много дивизоров $E$ с дискрепантностью а $\left(E, D^{\prime}\right)=-1$ и пара $\left(S, D^{\prime}\right)$ логканонична, то для $K_{S}+D$ существует 1-, 2- или 6-нелогтерминальное по Кавамате дополнение.

Из доказательства следствия 2.6 получаем, что дивизор $K_{S}+D^{\prime}$ имеет $\mathbb{Q}$-дополнение. Поэтому по теореме 2.3 [2] имеем требуемое.

\section{§3. Исключительные нерациональные логповерхности}

Tеорема 3.1 (ср. [3; предложение 9.2.2]). Пусть $\left(S, D=\sum d_{i} D_{i}\right)$ - проективная логповерхность, где $D$ - граница. Предположим, что выполнень следующие условия:

1) существует $\mathbb{Q}$-дополнение $\Theta=\sum \theta_{i} \Theta_{i}$ для $K_{S}+D$;

2) поверхность $S$ нерациональна и пара $(S, D)$ исключительна;

3) дивизор $\Theta \neq 0$ или $S$ имеет недювалевскую особенность. 
Тогда для $K_{S}+D$ существует 2-, 3-, 4- или 6-дополнение. Более того, имеем один из следующих двух случаев:

a) $S \cong C \times \mathbb{P}^{1}$, әде $C$ - эллиптическая кривая, а $D_{i}$ являются сечениями соответствующего $\mathbb{P}^{1}$-расслоения;

b) $S \cong \mathbb{P}_{C}(\mathscr{E})$, әде $\mathscr{E}$ - неразложимый пучок степени 1 на әллиптической кривой $C$, соответствующий расширению

$$
0 \longrightarrow \mathscr{O}_{C} \longrightarrow \mathscr{E} \longrightarrow \mathscr{O}_{C}(O) \longrightarrow 0
$$

тогда $D_{i} \sim 2 E-f^{*}\left(O+t_{i}\right)$ или $D_{i} \sim 4 E-2 f^{*} O$, где $E-$ сечение $f: S \rightarrow C$, а $t_{i}$ - әлемент порядка 2 в $\operatorname{Pic}(C)$.

ДоказАТЕльство. Пусть $\phi: \widetilde{S} \rightarrow S$ - минимальное разрешение. Тогда

$$
K_{\widetilde{S}}+\widetilde{\Theta}=\phi^{*}\left(K_{S}+\Theta\right) \equiv 0 .
$$

Условие 3$)$ дает, что $\widetilde{\Theta} \neq 0$, т.е. $\varkappa(\widetilde{S})=-\infty$. Пусть $S_{\min }-$ минимальная модель для $\widetilde{S}$. По условию $S_{\min }$ является минимальной линейчатой поверхностью над кривой $C$ с $p_{a}(C) \geqslant 1$. Образ дивизора $\widetilde{\Theta}$ на $S_{\min }$ обозначим через $\bar{\Theta}$. Если на $S_{\min }$ существует неприводимая кривая $E$ с $E^{2}<0$, то

$$
\left(K_{S_{\min }}+E\right) \cdot E=\left(-\sum_{i: \bar{\Theta}_{i} \neq E} \theta_{i} \bar{\Theta}_{i}\right) \cdot E+\varepsilon E^{2}<0,
$$

где $\varepsilon>0$. Отсюда $p_{a}(E)=0$ и имеем противоречие с $p_{a}(C) \geqslant 1$.

Поскольку $0 \geqslant 8-8 p_{a}(C)=K_{S_{\min }}^{2}=\bar{\Theta}^{2} \geqslant 0$, то $p_{a}(C)=1, \bar{\Theta}_{i}^{2}=\bar{\Theta}_{i} \bar{\Theta}_{j}=0$ для всех $i, j$. Так как $\theta_{i}<1$ для всех $i$, то пара $\left(S_{\min }, \bar{\Theta}\right)$ является терминальной. Поэтому $\mathbb{P}_{C}(\mathscr{E}) \cong S_{\min } \cong \widetilde{S} \cong S$, где $\operatorname{deg} \mathscr{E} \geqslant 0$. Из [12; гл. 5$]$ и [2; примеры $\left.1.1,2.1\right]$ получаем оставшиеся утверждения.

ЗАмечАниЕ 3.2 [2; пример 2.1]. В теореме 3.1 в случае b) линейная система $\left|4 E-2 f^{*} O\right|$ задает структуру эллиптического расслоения с тремя вырожденными (двукратными) слоями, которые линейно эквиваленты $2 E-f^{*}\left(O+t_{i}\right)$.

СлЕДСТВИЕ 3.3. В условиях теоремы 3.1 имеем $\delta(S, D)=0$.

\section{§4. Построение моделей для логповерхностей дель Пеццо с $\delta=0$}

Классификация исключительных логповерхностей с $\delta=1,2$ получена в работах [2], [9]. Исключительные нерациональные логповерхности полностью изучены в теореме 3.1. Таким образом, остается изучить последний оставшийся случай исключительные рациональные логповерхности с $\delta=0$.

ОПРЕДЕЛЕНИЕ 4.1. Пара $(S, D)$ называется логповерхностью дель Пеццо, где $D$ является границей, если выполнены следующие условия:

1) $-\left(K_{S}+D\right)$ - численно эффективньй дивизор;

2) $K_{S}+D-$ логканонический дивизор;

3) сушествует $\mathbb{Q}$-дополнение для $K_{S}+D$.

Рассмотрим предельную возможность. 
ПримеР 4.2 [13; замечание 1.2], [14; примеры 4.2, 5.3]. а) Пусть $\bar{S}=C \times$ $C$, где $C=\mathbb{C} /\left(\mathbb{Z}+\varepsilon_{3} \mathbb{Z}\right)$ - эллиптическая кривая и $\varepsilon_{3}$ - первообразный корень степени 3 из 1 . Групша $\mathbb{Z}_{3}$ действует на кривой $C$ умножением на $\varepsilon_{3}$. Тогда $S=$ $\bar{S} / \mathbb{Z}_{3}$ является поверхностью с $3 K_{S} \sim 0, \rho(S)=4$, и $\operatorname{sing} S$ состоит из девяти особенностей $\frac{1}{3}(1,1)$.

b) Пусть поверхность $\bar{S}=J(C)$ является якобианом гиперэллиптической кривой $C: y^{2}=x^{5}-1$ рода 2 . Группа $\mathbb{Z}_{5}$ порождена автоморфизмом $(x, y) \mapsto\left(\varepsilon_{5} x, y\right)$ кривой $C$, где $\varepsilon_{5}-$ первообразный корень степени 5 из 1 . Тогда $S=\bar{S} / \mathbb{Z}_{5}$ является поверхностью с $5 K_{S} \sim 0, \rho(S)=2$, и $\operatorname{Sing} S$ состоит из пяти особенностей $\frac{1}{5}(2,1)$.

c) Рассмотрим на $\mathbb{P}^{2}$ три неприводимые кривые: $\bar{E}_{1} \sim \mathscr{O}_{\mathbb{P}^{2}}(1), \bar{E}_{2} \sim \bar{E}_{3} \sim$ $\mathscr{O}_{\mathbb{P}^{2}}(4)$. Кривые $\bar{E}_{2}$ и $\bar{E}_{3}$ имеют по три обыкновенных двойных точки $Q_{5}, Q_{6}, Q_{7}$ и $Q_{8}, Q_{9}, Q_{10}$ соответственно. Прямая $\bar{E}_{1}$ пересекает кривые $\bar{E}_{2}$ и $\bar{E}_{3}$ в точках $Q_{1}$, $Q_{2}, Q_{3}$ и $Q_{4}$. Кривая $\bar{E}_{2}$ пересекает $\bar{E}_{3}$ в точках $Q_{1}, \ldots, Q_{10}$. Раздуем $\mathbb{P}^{2}$ в точках $Q_{1}, \ldots, Q_{10}$ и стянем собственњые прообразы кривых $\bar{E}_{1}, \bar{E}_{2}, \bar{E}_{3}$. Получим поверхность $\widetilde{S}$ с $3 K_{\widetilde{S}} \sim 0, \rho(\widetilde{S})=8$, и $\operatorname{Sing} \widetilde{S}$ состоит из трех особенностей $\frac{1}{3}(1,1)$. Стягивая $(-2)$-кривые на $\widetilde{S}$, получаем поверхность $S$ с $3 K_{S} \sim 0$ и с тремя недювалевскими особенностями $\frac{1}{3}(1,1)$.

ТЕОРема 4.3. Пусть $S$ - рациональная исключительная логповерхность дель Пецчо, $D=0$. Предположим, что $a(E, 0)>-\frac{1}{2}$ для всех $E$ и не существует $\mathbb{Q}$-дополнения $\Theta=\sum \frac{1}{2} \Theta_{i}$ для $K_{S}$. Тогда $S$ является поверхностью из примера 4.2 .

ДоКАЗАТЕЛЬСТво. Поверхность $S$ должна иметь недювалевскую особенность, иначе $h^{0}\left(S, \mathscr{O}_{S}\left(-K_{S}\right)\right) \geqslant 1$.

ЛЕмма 4.4. Пусть $(X \ni P)$ - двумерная, недювалевская особенность. Предположим, что

$$
M=\min \{a(E, 0) \mid E-\text { исключительныц дивизор }\}>-\frac{1}{2} .
$$

Тогда $(X \ni P) \cong_{\mathrm{an}}\left(\mathbb{C}^{2} \ni 0\right) / \mathbb{Z}_{2 n+1}(n, 1)$, где $n \geqslant 1$. В частности, $M=$ $-\frac{n}{2 n+1} \leqslant-\frac{1}{3}$.

ДокАЗАтЕльСтво. Если $(X \ni P)$ - нециклическая особенность, то раздутие центральной вершины графа минимального разрешения дает дискрепантность меньше или равную $-\frac{1}{2}$. Поэтому $(X \ni P)$ является циклической особенностью. Пусть $\widetilde{X} \rightarrow X$ является раздутием с единственной исключительной кривой $E$, для которой индекс самопересечения $k$ на минимальном разрешении $(X \ni P)$ не больше -3 . Тогда

$$
\begin{aligned}
-\frac{1}{2} & <a(E, 0)=-1+\frac{-2+\operatorname{Diff}_{E}(0)}{E^{2}} \\
& =-1+\frac{-2+\left(m_{1}-1\right) / m_{1}+\left(m_{2}-1\right) / m_{2}}{-k+q_{1} / m_{1}+q_{2} / m_{2}} .
\end{aligned}
$$

Отсюда $\left(m_{1}, q_{1}\right)=(1,0),\left(m_{2}, q_{2}\right)=\left(m_{2}, m_{2}-1\right), k=3$. 
Пусть $P_{1}, \ldots, P_{r}$ - недювалевские особенности $S$ типов

$$
\frac{1}{2 n_{1}+1}\left(n_{1}, 1\right), \ldots, \frac{1}{2 n_{r}+1}\left(n_{r}, 1\right) .
$$

Пусть $f: \widetilde{S} \rightarrow S$ - минимальное разрешение. Тогда $K_{\widetilde{S}}+\Delta=f^{*} K_{S}$. По лемме 4.4

$$
h^{2}\left(\widetilde{S}, \mathscr{O}_{\widetilde{S}}\left(-2 K_{\widetilde{S}}-\lfloor 3 \Delta\rfloor\right)\right)=h^{0}\left(\widetilde{S}, \mathscr{O}_{\widetilde{S}}\left(3 K_{\widetilde{S}}+\lfloor 3 \Delta\rfloor\right)\right)=0,
$$

кроме случая $n_{1}=\cdots=n_{r}=1$ и $K_{S} \equiv 0$. Найдем оставшиеся возможности для $n_{1}, \ldots, n_{r}$. По теореме Римана-Роха и формуле Нётера имеем систему

$$
\left\{\begin{aligned}
& 0=h^{0}\left(\widetilde{S}, \mathscr{O}_{\widetilde{S}}\left(-2 K_{\widetilde{S}}-\lfloor 3 \Delta\rfloor\right)\right)= 3 K_{S}^{2}+r+1-3 \cdot \sum_{i=1}^{r} \frac{n_{i}}{2 n_{i}+1} \\
&+h^{1}\left(\widetilde{S}, \mathscr{O}_{\widetilde{S}}\left(-2 K_{\widetilde{S}}-\lfloor 3 \Delta\rfloor\right)\right) ; \\
& K_{S}^{2}-\sum_{i=1}^{r} \frac{n_{i}}{2 n_{i}+1}+\rho(S)+n_{1}+\cdots+n_{r} \leqslant 10 .
\end{aligned}\right.
$$

Лемма 4.5 [15; следствие 9.2]. Пусть $X$ - рациональная поверхность с логтерминальными особенностями и с $\rho(X)=1$. Тогда

$$
\sum_{P \in \operatorname{Sing} X} \frac{m_{P}-1}{m_{P}} \leqslant 3
$$

где $m_{P}-$ порядок локальной фундаментальной группь $\pi_{1}\left(U_{P} \backslash\{P\}\right), U_{P}-$ достаточно малая окрестность точки $P$.

Учитьвая, что $K_{S}^{2} \geqslant 0$, и лемму 4.5 , из системы получаем $K_{S}^{2}=0, \rho(S)=2$, $n_{1}=\cdots=n_{5}=2, \operatorname{Sing} S=\left\{P_{1}, \ldots, P_{5}\right\}$. Более того, имеем $K_{S} \equiv 0$. Действительно, пусть $K_{S} \not \equiv 0$. Если существует кривая $E$ на $S$ с $E^{2}<0$, то после ее стягивания имеем противоречие с леммой 4.5. Поэтому имеем $\mathbb{P}^{1}$-расслоение (в общей точке) $S \rightarrow Z$, но это невозможно из-за классификации таких расслоений [3; теорема 7.1.12].

Пусть $\bar{S} \rightarrow S$ - каноническое накрытие. Имеем два случая [13; теорема С].

А) Пусть $\bar{S}$ является абелевой поверхностью. Тогда из теоремы C [13] следует, что $S$ является поверхностью из примера $4.2, \mathrm{a}), \mathrm{b})$. При этом $n_{1}=\cdots=n_{9}=1$ или $n_{1}=\cdots=n_{5}=2$.

В) Пусть $\bar{S}$ является К3-поверхностью. Тогда из [14; теорема 5.1] получаем, что $n_{1}=n_{2}=n_{3}=1$. Ясно, что одна из минимальных моделей поверхности $\widetilde{S}$ будет $S_{\text {min }} \cong \mathbb{P}^{2}$. Пусть $\varphi: \widetilde{S} \rightarrow S_{\min }-$ соответствующий бирациональный морфизм. Положим $\bar{\Delta}=\varphi(\Delta)=\frac{1}{3}\left(\varphi\left(E_{1}\right)+\varphi\left(E_{2}\right)+\varphi\left(E_{3}\right)\right)$, где $E_{i}$ является исключительной кривой над точкой $P_{i}$. Пусть $\varphi$ стягивает кривую $E$, отличную от $E_{i}$ для всех $i$. Тогда пара $\left(S_{\min } \ni \bar{P}, \bar{\Delta}\right)$ будет канонической, где $\bar{P}=\varphi(E)$. Очевидно, что пара $\left(S_{\min } \ni \bar{P}, \bar{\Delta}\right)$ аналитически изоморфна $\left(\mathbb{C}^{2} \ni 0, \frac{1}{3}\left\{x^{3}+y^{l}=0\right\}\right)$, где $l=3,4$, или $\left(\mathbb{C}^{2} \ni 0, \frac{1}{3}\left\{x^{3}+x y^{3}=0\right\}\right)$, или $\left(\mathbb{C}^{2} \ni 0, \frac{1}{3}\left\{x^{2} y+y^{4}=0\right\}\right)$. 
Лемма 4.6. Существует поверхность $S^{\prime}$, әде $\varphi: S \stackrel{\psi}{\longrightarrow} S^{\prime} \longrightarrow S_{\min }$, на которой $\psi\left(E_{i}\right)$ будет $(+1)$-неособой рациональной кривой для некоторого $i$.

ДокаЗАтЕльство. Если $\bar{\Delta}=\frac{1}{3} \bar{E}_{j}$, то $p_{a}\left(\bar{E}_{j}\right)=28$. Кривая $\bar{E}_{j}$ должна иметь две точки кратности 4 и восемь точек кратности 3 , но это невозможно. Поэтому существует кривая $\bar{E}_{j}$ такая, что $\left(\mathscr{O}_{\mathbb{P}^{2}}(5)-\bar{E}_{j}\right)$ - численно эффективный дивизор. После раздутия особенностей кривой $\bar{E}_{j}$ получаем кривую с индексом самопересечения большим или равным +1 , что и требовалось доказать.

Можно, считать, что линейная система $\left|\psi\left(E_{i}\right)\right|$ задает бирациональный морфизм $S^{\prime} \rightarrow S_{\min }[9 ;$ предложение 1.10$]$ и $\bar{\Delta}=\frac{1}{3} \bar{E}_{1}+\frac{1}{3} \bar{E}_{2}+\frac{1}{3} \bar{E}_{3}$.

С помошью перебора всех вариантов для $\bar{E}_{1}, \bar{E}_{2}, \bar{E}_{3}$ на $S_{\min } \cong \mathbb{P}^{2}$ легко показать, что на $\widetilde{S}$ существует четыре попарно непересекающиеся $(-1)$-кривые, каждая из которых пересекает все $E_{i}$. Пусть $S^{\prime}$ получается из $\widetilde{S}$ их стягиванием. Тогда имеем случай с) из примера 4.2 .

Классификация логповерхностей дель Пецо очень важна для изучения трехмерных экстремальных стягиваний и особенностей, так как существует индукция от (локального) трехмерного стягивания к двумерному логмногообразию [2], $[4],[8]$. Напомним, что в результате индукции получаются логповерхности $(S, D)$ такие, что дивизор $-\left(K_{S}+D\right)$ численно эффективен и объемен, а коэффициенты $D$ являются стандартными. Для того чтобы получить эффективную классификацию, исключительные логповерхности дель Пецо с $\delta=1,2$ рассматриваются в более широком классе коэффициентов $-\Phi_{\mathrm{m}}[2],[9]$. При этом условие объемности заменяется, например, на требование существования $\mathbb{Q}$-дополнения для $K_{S}+D$. Последнее позволяет получить классификацию логповерхностей Энриквеса с $\delta=1,2[16],[17]$. Поэтому в случае $\delta=0$ множество $\Phi_{\mathrm{sm}}$ будет расширено до $\Phi_{i}$. Сейчас основная цель - это построить модели с числом Пикара равным 1 или 2 для $(S, D)$.

ОПРЕДЕЛЕНИЕ 4.7. Пусть $(S, D)$ - исключительная логповерхность дель Пеццо с $\delta(S, D)=0$, где $S$ - рациональная поверхность. Тогда пара $(S, D)$ типа $\Phi_{i}$ называется исключительной логповерхностью дель Пецио типа $\Phi_{i}$, где $i=$ $2,3,4,5,6$.

Рассмотрим три случая.

Случай І. Пусть $i=3,4,5,6$. Положим

$$
\widehat{D}=\sum_{d_{k} \geqslant(i-1) / i} c\left(D_{k}\right) D_{k}+\sum_{d_{k}<(i-1) / i} d_{k} D_{k}
$$

где $c\left(D_{k}\right)=c\left(S, D-d_{k} D_{k} ; D_{k}\right)$ - логканонический порог дивизора $D_{k}$ для логпары $\left(S, D-d_{k} D_{k}\right)$. По предложению 1.15 дивизор $K_{S}+\widehat{D}$ является логканоническим. По следствию 2.6 дивизор $-\left(K_{S}+\widehat{D}\right)$ не является численно эффективньм. Предположим, что $\rho(S) \geqslant 3$. Тогда сушествует исключительная кривая $E$ с $\left(K_{S}+\widehat{D}\right) \cdot E>0$ (см. [2; доказательство теоремы 4.1]). Пусть $\varphi: S \rightarrow S^{\prime}$ - это стягивание $E$. В отличие от случая $\delta(S, D) \geqslant 1$ бирациональный морфизм $\varphi$ может стянуть кривую из $D$ с коэффициентом больше или равным $\frac{i-1}{i}$. 
Лемма 4.8. Пусть $D=\alpha E+D^{\circ}, \alpha \geqslant \frac{i-1}{i}$ и $P=\varphi(E)$. Тогда имеем один из следующих случаев:

1) $\left(S^{\prime} \ni P, \varphi(D)\right) \cong_{\text {an }}\left(\mathbb{C}^{2} \ni 0, \frac{1}{2}\{x=0\}+\left(\frac{3}{4}+\varepsilon\right)\left\{x+y^{3}=0\right\}\right)$, әде $\varepsilon<\frac{1}{60}$ и $i=4$, морфизм $\varphi$ является взвешенным раздутием с весами $(3,1)$;

2) $\left(S^{\prime} \ni P, \varphi(D)\right) \cong_{\text {an }}\left(\mathbb{C}^{2} \ni 0,\left(\frac{2}{3}+\varepsilon_{1}\right)\{x=0\}+\left(\frac{2}{3}+\varepsilon_{2}\right)\left\{x+y^{2}=0\right\}\right)$, где $\varepsilon_{1}+\varepsilon_{2}<\frac{1}{24}$ и $i=3$, морфизм $\varphi$ является взвешенным раздутием с весами $(2,1)$;

3) $\left(S^{\prime} \ni P, \varphi(D)\right) \cong$ an $\left(\mathbb{C}^{2} \ni 0, \frac{1}{2}\{x=0\}+\left(\frac{2}{3}+\varepsilon\right)\left\{x+y^{l}=0\right\}\right)$, əде $l=3,4$, $i=3$ и $\varepsilon<\frac{3}{4 l}-\frac{1}{6}$, морфизм $\varphi$ является взвешенныцм раздутием с весами $(l, 1)$

4) Пусть $\left(S^{\prime} \ni P, \varphi(D)\right) \cong_{\text {an }}\left(\mathbb{C}^{2} \ni 0,\left(\frac{i-1}{i}+\varepsilon\right)\left\{x^{2}+y^{3}=0\right\}\right)$, где $i=5,4,3$, $\varepsilon<\frac{1}{180}$ при $i=5, \varepsilon<\frac{1}{20}$ при $i=4, \varepsilon<\frac{1}{12}$ при $i=3$, морфизм $\varphi$ является взвешенныц раздутием с весами $(3,2)$.

ДокАЗАТЕЛЬство. Заметим, что $E$ должна пересекаться с кривой из $D^{\circ}$ с коэффициентом больше или равным $\frac{i-1}{i}$. Рассмотрим случай, когда $i=3$ и на $E$ сушествует точка из п. 2) предложения 1.15 при $k=3$. Тогда

$\left(K_{S}+D\right) \cdot E>\left(K_{S}+E+D^{\circ}\right) \cdot E=-2+\operatorname{deg} \operatorname{Diff}_{E}(0)+D^{\circ} \cdot E \geqslant-2+\frac{3}{2}+\frac{2}{3}>0$.

Противоречие. Поэтому $E$ имеет коэффициент 1 в $\widehat{D}$. Имеем три возможности.

i) Предположим, что $\operatorname{Diff}_{E}(0)=0$. Тогда $\left(S^{\prime} \ni P\right) \cong_{\text {an }}\left(\mathbb{C}^{2} \ni 0\right) / \mathbb{Z}_{n}(1,1)$. Поскольку $\left(K_{S}+\widehat{D}\right) \cdot E>0$ и $\left(K_{S}+D\right) \cdot E \leqslant 0$, то простейшие вычисления показывают, что возможность не реализуется.

ii) Предположим, что $\operatorname{Diff}_{E}(0)=\frac{k-1}{k} P$, где $k \geqslant 2$. Рассмотрим, например, случай $i=3$. Поскольку $\operatorname{deg} \operatorname{Diff}_{E}\left(D^{\circ}\right)<2$ и $\operatorname{deg} \operatorname{Diff}_{E}(\widehat{D}-E)>2$, то $k=2,3,4,5$ и $P$ - неособая точка $S^{\prime}$. Более того, получаем

a) $\operatorname{Diff}_{E}\left(D^{\circ}\right)=\frac{1}{2} P+\left(\frac{2}{3}+\varepsilon_{1}\right) P_{1}+\left(\frac{2}{3}+\varepsilon_{2}\right) P_{2}$ или

b) $\operatorname{Diff}_{E}\left(D^{\circ}\right)=\frac{k-1}{k} P+\frac{1}{2} P_{1}+\left(\frac{2}{3}+\varepsilon\right) P_{2}$, где $k=3,4,5$.

Остается проверить, что $-a(E, \varphi(D))<\frac{i}{i+1}$. Получаем случаи 2$)$ и 3$)$.

iii) Предположим, что

$$
\operatorname{Diff}_{E}(0)=\frac{k_{1}-1}{k_{1}} P_{1}+\frac{k_{2}-1}{k_{2}} P_{2},
$$

где $k_{1}, k_{2} \geqslant 2$. Поскольку $\operatorname{deg} \operatorname{Diff}_{E}\left(D^{\circ}\right)<2$, то можно считать, что $k_{1}=2$. Аналогичные вычисления дают случай 4$)$.

Случай II. При $i=4,5,6$ для поверхности $S^{\prime}$ повторим вышеприведенную процедуру. При $i=4$ случай, когда на исключительной кривой лежит точка из п. 1) леммы 4.8, отсекается, как и случай 2 ) из предложения 1.15 при $k=3$ (см. доказательство леммы 4.8). В результате придем к поверхности $\bar{S}$ с $\rho(\bar{S})=1$ или к поверхности $\bar{S}$ с $\rho(\bar{S})=2$ и со структурой $\mathbb{P}^{1}$-расслоения.

Пусть теперь $i=3$. Положим $D^{\prime}=\sum d_{k}^{\prime} D_{k}^{\prime}=\varphi(D)$. Повторим вышеприведенную процедуру. Дивизор $\widehat{D}^{\prime}$ определяется как и дивизор $\widehat{D}$, но если на $D_{k}^{\prime}$ лежит точка из п. 2) леммы 4.8 , то положим $c\left(D_{k}^{\prime}\right)=\frac{3}{4}$. 
Пусть $\varphi^{\prime}: S^{\prime} \rightarrow S^{\prime \prime}$ - это стягивание кривой $E^{\prime}$ из $D^{\prime}$ с коэффициентом больше или равным $\frac{2}{3}$. Появились два новых случая.

i) Как и ранее, случай, когда на $E^{\prime}$ лежит точка из п. 3) леммы 4.8 , невозможен.

ii) Предположим, что на $E^{\prime}$ лежит точка из п. 2) леммы 4.8. Тогда докажем, что $\operatorname{Diff}_{E^{\prime}}\left(\widehat{D}^{\prime}-\frac{3}{4} E^{\prime}\right) \neq \frac{3}{2} Q$, где $Q$ - точка на $E^{\prime}$. Действительно, в противном случае имеем

$$
\left\{\begin{aligned}
\left(K_{S^{\prime}}+\widehat{D}^{\prime}\right) \cdot E^{\prime} & =\left(K_{S^{\prime}}+\frac{3}{4} E^{\prime}+\frac{3}{4} D_{2}^{\prime}\right) \cdot E^{\prime}=-\frac{1}{2}-\frac{1}{4} E^{\prime 2}>0 \\
\left(K_{S^{\prime}}+D^{\prime}\right) \cdot E^{\prime} & =\left(K_{S^{\prime}}+\left(\frac{2}{3}+\varepsilon_{1}\right) E^{\prime}+\left(\frac{2}{3}+\varepsilon_{2}\right) D_{2}^{\prime}\right) \cdot E^{\prime} \\
& =-\frac{2}{3}+2 \varepsilon_{2}-\left(\frac{1}{3}-\varepsilon_{1}\right) E^{\prime 2} \leqslant 0 .
\end{aligned}\right.
$$

Так как $\varepsilon_{1}+\varepsilon_{2}<\frac{1}{24}$, то система неравенств противоречива. Простые вычисления показывают, что возможен случай

$$
\left(S^{\prime \prime} \ni P^{\prime}, \varphi^{\prime}\left(D^{\prime}\right)\right) \cong \text { an }\left(\mathbb{C}^{2} \ni 0,\left(\frac{2}{3}+\varepsilon\right)\left\{x^{2}+y^{5}=0\right\}\right),
$$

где $\varepsilon<\frac{1}{120}, P^{\prime}=\varphi^{\prime}\left(E^{\prime}\right)$ и $\varphi^{\prime}$ является взвешенным раздутием с весами $(2,1)$. Повторяя процедуру, мы придем к вышеописанной поверхности $\bar{S}$.

Случай III. Пусть $i=2$. Сейчас основная задача состоит в том, как правильно увеличить дивизор $D=\sum d_{l} D_{l}$ до дивизора $\widehat{D}=\sum c_{l} D_{l}$, где $c_{l} \geqslant d_{l}$ для всех $l$. После этого увеличения дивизор $K_{S}+\widehat{D}$ должен быть логканоническим, но не логтерминальным по Кавамате. Далее, как и прежде, найдется исключительная кривая $E \mathrm{c}\left(K_{S}+D\right) \cdot E>0$ при условии $\rho(S) \geqslant 3$. Соответствующий морфизм обозначим через $\varphi: S \rightarrow S^{\prime}$.

Опишем построение $\widehat{D}$. Пусть $D_{t}-$ неособая кривая. Если на $D_{t}$ найдется точка из пा. 1) или 2) предложения 1.15 при $k \geqslant 4$, то $E \neq D_{t}$. Действительно, иначе имеем

$$
\left(K_{S}+D\right) \cdot D_{t}>\left(K_{S}+D_{t}+\sum_{l \neq t} d_{l} D_{l}\right) \cdot D_{t} \geqslant-2+\left(\sum_{l \neq t} d_{l} D_{l}\right) \cdot D_{t} \geqslant 0
$$

Поэтому в этом случае неважно, насколько надо увеличить коэффициент $d_{t}$.

Рассмотрим остальные случаи. Если на $D_{t}$ найдется точка из пा. 1) или 2) предложения 1.15 при $k=3$, то положим $c_{t}=\frac{2}{3}$. Если на $D_{t}$ найдется точка из пा. 1) или 2) предложения 1.15 при $k=2$, то положим $c_{t}=\frac{3}{4}$.

Во всех остальных случаях произвольным образом увеличим все оставшиеся коэффициенты $d_{l}$ до максимально возможных значений.

Лемма 4.9. Пусть $D=\alpha E+D^{\circ}, \alpha \geqslant \frac{1}{2} u P=\varphi(E)$. Тогда имеем один из следующих случаев:

1) $\left(S^{\prime} \ni P, \varphi(D)\right) \cong$ an $\left(\mathbb{C}^{2} \ni 0,\left(\frac{1}{2}+\varepsilon\right)\left\{x^{3}+y^{4}=0\right\}\right)$, әде $\varepsilon<\frac{1}{24}$, морфизм $\varphi$ является взвешенным раздутием с весами $(1,1)$; 
2) $\left(S^{\prime} \ni P, \varphi(D)\right) \cong$ an $\left(\mathbb{C}^{2} \ni 0,\left(\frac{1}{2}+\varepsilon_{1}\right)\{y=0\}+\left(\frac{1}{2}+\varepsilon_{2}\right)\left\{x^{2}+y^{3}=0\right\}\right)$, где $3 \varepsilon_{1}+\varepsilon_{2}<\frac{1}{4}$, морфизм $\varphi$ является взвешенным раздутием с весами $(1,1)$

3) $\left(S^{\prime} \ni P, \varphi(D)\right) \cong$ an $\left(\mathbb{C}^{2} \ni 0,\left(\frac{1}{2}+\varepsilon\right)\left\{x^{2}+y^{2 k+1}=0\right\}\right)$, әде $\varepsilon<\frac{3}{8 k+4}$, морфизм $\varphi$ является взвешенньц раздутием с весами $(k, 1)$;

4) $\left(S^{\prime} \ni P, \varphi(D)\right) \cong_{\text {an }}\left(\mathbb{C}^{2} \ni 0,\left(\frac{1}{2}+\varepsilon_{1}\right)\{x=0\}+\left(\frac{1}{2}+\varepsilon_{2}\right)\{y=0\}+\right.$ $\left.\left(\frac{1}{2}+\varepsilon_{3}\right)\{x+y=0\}\right)$, где $\varepsilon_{1}+\varepsilon_{2}+\varepsilon_{3}<\frac{1}{6}$, морфизм $\varphi$ является взвешенным раздутием с весами $(1,1)$;

5) $\left(S^{\prime} \ni P, \varphi(D)\right) \cong$ an $\left(\mathbb{C}^{2} \ni 0,\left(\frac{1}{2}+\varepsilon_{1}\right)\{x=0\}+\left(\frac{1}{2}+\varepsilon_{2}\right)\left\{x+y^{k}=0\right\}\right)$, где $\varepsilon_{1}+\varepsilon_{2}<\frac{2}{3 k}$, морфизм $\varphi$ является взвешенным раздутием с весами $(k, 1)$.

ДокАЗАТЕЛЬСТво. Поскольку дивизор $K_{S}+D$ является $\frac{1}{2}$-логтерминальным, то особые точки $S$, лежашие на $E$, - это дювалевские особенности типа $\mathbb{A}_{n}$ (см. предложение 1.15). По той же причине на кривой $E$ лежит не более одной особой точки.

Предположим, что $E$ имеет точку касания кратности 3 с кривой $D_{1}$ из $D^{\circ}$. Так как $\left(K_{S}+D\right) \cdot E \leqslant 0$, то $\operatorname{Diff}_{E}\left(D^{\circ}-d_{1} D_{1}\right)=0$ и получаем случай 1$)$.

Если $E$ имеет точку касания кратности 2 с кривой $D_{1}$ из $D^{\circ}$, то, поступая как и в лемме 4.8 , получаем случаи 2 ) и 3 ).

Для оставшейся возможности получаем случаи 4) и 5).

Повторим вышеописанную процедуру. В результате многократного повторения, как и в случае II, могут появиться две новые особенности:

$$
\left(\mathbb{C}^{2} \ni 0,\left(\frac{1}{2}+\varepsilon_{1}\right)\{y=0\}+\left(\frac{1}{2}+\varepsilon_{2}\right)\{x=0\}+\left(\frac{1}{2}+\varepsilon_{3}\right)\left\{x+y^{k}=0\right\}\right),
$$

где $\varepsilon_{1}+k\left(\varepsilon_{2}+\varepsilon_{3}\right)<\frac{1}{6}$, и

$$
\left(\mathbb{C}^{2} \ni 0,\left(\frac{1}{2}+\varepsilon_{1}\right)\{y=0\}+\left(\frac{1}{2}+\varepsilon_{2}\right)\left\{x^{2}+y^{2 k+1}=0\right\}\right)
$$

где $\varepsilon_{1}+2 k \varepsilon_{2}<\frac{1}{6}$.

Все вышесказанное позволяет дать определение модели для исключительной логповерхности дель Пещо типа $\Phi_{i}($ cp. $[2 ; \S 5])$.

ОПРЕДЕЛЕНИЕ 4.10. Пусть $(S, D)$ является исключительной логповерхностью дель Пещц типа $\Phi_{i}, i=2,3,4,5,6$, за исключением следующих точек (при $i=6$ исключений нет).

1) При $i=5$ см. п. 4) леммы 4.8 .

2) При $i=4$ см. пाп. 1), 4) леммы 4.8 .

3) При $i=3$ см. пп. 2), 3), 4) леммы 4.8, а также (I) из случая II.

4) При $i=2$ см. пा. 1$), 3), 5)$ леммы 4.9 , но возможно с другими ограничениями на значения $\varepsilon, \varepsilon_{1}, \varepsilon_{2}$, а также (II) и (III) из случая III. 
Тогда логпара $(S, D)$ назьвается моделью типа $\Phi_{i}$, если выполнено одно из следующих двух условий:

A) $\rho(S)=1$;

В) $\rho(S)=2$, конус $\overline{\mathrm{NE}}(S)$ порожден двумя экстремальными лучами $R_{1}$ и $R_{2}$; луч $R_{1}$ задает $\mathbb{P}^{1}$-расслоение (в общей точке); если луч $R_{2}$ задает бирациональное стягивание кривой $E$, то $E$ является компонентой дивизора $D$ с коэффициентом больше или равным $\frac{i-1}{i}$.

ЗАмечание 4.11. Заметим, что в определении модели типа $\Phi_{i}$ условие $\frac{1}{i}$-логтерминальности дивизора $K_{S}+D$ нарушается только в неособых точках поверхности $S$.

ЗАмЕчАниЕ 4.12. Важная задача заключается в классификации моделей типа $\Phi_{i}$. После завершения классификации моделей типа $\Phi_{2}$ остается описать такие исключительные поверхности $S$, что дивизор $K_{S}$ является $\frac{1}{2}$-логтерминальньм и для него существует 2 -дополнение $\Theta=\sum \frac{1}{2} \Theta_{i}$ (см. теорему 4.3). Это завершит классификацию исключительных логповерхностей дель Пецю (см. [2], [9]) и позволит завершить описание логповерхностей Энриквеса (см. [16], [17]).

ЗАмЕчАниЕ 4.13. Согласно недавно полученным результатам можно отказаться от условия сушествования $\mathbb{Q}$-дополнения в теоремах $2.1,3.1$ и 4 [18].

\section{Список литературы}

1. Шокуров В. В. Трехмерные логперестройки // Изв. АН СССР. Сер. матем. 1992. Т. 56. № 1. C. $105-203$.

2. Shokurov V. V. Complements on surfaces // J. Math. Sci. (New York). 2000. V. 102. № 2. P. 3876-3932.

3. Prokhorov Yu. G. Lectures on complements on log surfaces. Tokyo: Math. Soc. Japan, 2001. (MSJ Memoirs. V. 10.)

4. Прохоров Ю. Г., Шокуров В. В. Первая основная теорема о дополнениях: от глобального к локальному // Изв. АН СССР. Сер. матем. 2001. Т. 65. №6. С. 99-128.

5. Кудрявцев С.А. Классификация трехмерных исключителных логканонических гиперповерхностных особенностей. I // Изв. РАН. Сер. матем. 2002. Т. 66. № 5. С. 83-170.

6. Кудрявцев С. А. Классиффикация трехмерных исключительных логканонических гиперповерхностных особенностей. II // Изв. РАН. Сер. матем. 2004. Т. 68. № 2. С. 137-146.

7. Prokhorov Yu. G. Mori conic bundles with a reduced log terminal boundary // J. Math. Sci. (New York). 1999. V. 91. № 1. P. 1051-1059.

8. Prokhorov Yu. G. Boundedness of non-birational extremal contractions // Internat. J. Math. 2000. V. 11. №3. P. 393-411.

9. Кудрявєев С. А. Классификация исключительных логповерхностей дель Пеццо с $\delta=1$ // Изв. РАН. Сер. матем. 2003. Т. 67. №3. С. 45-78.

10. Kollar $J$. (ed.) Flips and abundance for algebraic threefolds. A summer seminar at the University of Utah, Salt Lake City, 1991. Paris: Soc. Math. France, 1992. (Astérisque. V. 211.)

11. Кудрявщев С. А. О чисто логтерминальных раздутиях // Матем. заметки. 2001. Т. 69. №6. C. 892-899.

12. Хартсхорн Р. Алгебраическая геометрия. М.: Мир, 1981.

13. Blache $R$. The structure of 1.c. surfaces of Kodaira dimension zero // J. Algebraic Geom. 1995. V. 4. P. $137-179$.

14. Zhang D.-Q. Logarithmic Enriques surfaces // J. Math. Kyoto Univ. 1991. V. 31. P. $419-466$. 
15. Keel S., McKernan J. Rational curves on quasi-projective surfaces. Providence, RI: Amer. Math. Soc., 1999. (Mem. Amer. Math. Soc. V. 669.)

16. Кудрявцев С.A. Классификация логповерхностей Энриквеса с $\delta=1 / /$ math.AG/ 0207270.

17. Кудрявцев C. А. Классификация логповерхностей Энриквеса с $\delta=2 / /$ Матем. заметки. 2002. Т. 72. № 5. С. 715-722.

18. Fedorov I. Yu., Kudryavtsev S. A. Q-complements on log surfaces // math. AG/0311325.

Московский государственнй университет

Поступила в редакцию

им. М.В. Ломоносова

01.07 .2003

E-mail: kudryav@mech.math.msu.su 